\title{
Quantum process tomography of a universal entangling gate implemented with Josephson phase qubits
}

\author{
R. C. Bialczak, M. Ansmann, M. Hofheinz, E. Lucero, M. Neeley, A. D. O'Connell, D. Sank, H. Wang, \\ J. Wenner, M. Steffen ${ }^{\dagger}$, A. N. Cleland and J. M. Martinis ${ }^{\star}$
}

Quantum gates must perform reliably when operating on standard input basis states and on complex superpositions thereof. Experiments using superconducting qubits have validated truth tables for particular implementations of, for example, the controlled-NOT gate ${ }^{1,2}$, but have not fully characterized gate operation for arbitrary superpositions of input states. Here we demonstrate the use of quantum process tomography, ${ }^{3,4}$ (QPT) to fully characterize the performance of a universal entangling gate between two superconducting qubits. Process tomography permits complete gate analysis, but requires precise preparation of arbitrary input states, control over the subsequent qubit interaction and ideally simultaneous single-shot measurement of output states. In recent work, it has been proposed to use QPT to probe noise properties ${ }^{5}$ and time dynamics ${ }^{6}$ of qubit systems and to apply techniques from control theory to create scalable qubit benchmarking protocols ${ }^{7,8}$. We use QPT to measure the fidelity and noise properties $^{5}$ of an entangling gate. In addition to demonstrating a promising fidelity, our entangling gate has an on-to-off ratio of 300 , a level of adjustable coupling that will become a requirement for future high-fidelity devices. This is the first solid-state demonstration of QPT in a two-qubit system, as QPT has previously been demonstrated only with single solid-state qubits ${ }^{9-11}$.

Universal quantum gates are the key elements in a quantum computer, as they provide the fundamental building blocks for encoding complex algorithms and operations. Single-qubit rotations together with the two-qubit controlled-NOT (CNOT) are known to provide a universal set of gates ${ }^{12}$. Here, we present the complete characterization of a universal entangling gate, the square root of $i$-SWAP (SQiSW; ref. 13), from which gates such as the CNOT can be constructed. The pulse sequence for a CNOT can be written in terms of a SQiSW gate as follows: CNOT $=R_{y}^{\mathrm{A}}\left(-90^{\circ}\right)\left[R_{x}^{\mathrm{A}}\left(90^{\circ}\right) \otimes\right.$ $\left.R_{x}^{\mathrm{B}}\left(-90^{\circ}\right)\right] \mathrm{SQ} i \mathrm{SW} R_{x}^{\mathrm{A}}\left(180^{\circ}\right) \mathrm{SQ} i \mathrm{SW} R_{y}^{\mathrm{A}}\left(90^{\circ}\right)$, where $R_{i}^{\alpha}(\theta)$ is a rotation about axis $j=x, y, z$ by an angle $\theta$ on qubit $\alpha=\mathrm{A}, \mathrm{B}$. The SQiSW is a 'natural' two-qubit gate, as it directly results from capacitive coupling of superconducting qubits, yielding qubit coupling of the general form $\sigma_{\mathrm{A} x} \sigma_{\mathrm{B} x}$ or $\sigma_{\mathrm{A} y} \sigma_{\mathrm{B} y}$, where $\sigma_{x, y}$ are the Pauli spin operators for qubits A and B (refs 14,15). Under the rotating-wave approximation, the corresponding interaction Hamiltonian has the form $H_{\text {int }}=\hbar(g / 2)(|01\rangle\langle 10|+| 10\rangle\langle 01|)$, where $|01\rangle=|0\rangle_{\mathrm{A}} \otimes|1\rangle_{\mathrm{B}}$ and $g$ is the coupling strength that depends on design parameters.

When the two qubits are placed on-resonance, the two-qubit states are coupled by $H_{\text {int }}$ as shown in Fig. 1a. The amplitudes of these states then oscillate in time, as described (in the rotating frame) by the unitary transformation

$$
U_{\mathrm{int}}=\left[\begin{array}{cccc}
1 & 0 & 0 & 0 \\
0 & \cos (g t / 2) & -i \sin (g t / 2) & 0 \\
0 & -i \sin (g t / 2) & \cos (g t / 2) & 0 \\
0 & 0 & 0 & 1
\end{array}\right]
$$

where $t$ is the interaction time and the representation is in the two-qubit basis set $\{|00\rangle,|01\rangle,|10\rangle,|11\rangle\}$. For an interaction time $g t=\pi$, the state amplitudes are swapped, such that $|01\rangle \rightarrow-i|10\rangle$ and $|10\rangle \rightarrow-i|01\rangle$. The SQiSW gate is formed by coupling for one-half this time, $g t=\pi / 2$, producing cosine and sine matrix elements with equal magnitudes, thus entangling the qubits. When the qubits are off-resonance by an energy $|\Delta| \gg g$ (Fig. 1b), the off-diagonal elements in $U_{\text {int }}$ are small and have average amplitude $g / \Delta$, effectively turning off the qubit-qubit interaction.

The electrical circuit for the capacitively coupled Josephson phase qubits ${ }^{16,17}$ used in this experiment is shown in Fig. 1c. Each phase qubit is a nonlinear resonator built from a Josephson inductance and an external shunting capacitance. When biased close to the critical current, the junction and its parallel loop inductance $L$ give rise to a cubic potential with energy eigenstates that are unequally spaced. The two lowest levels are used as the qubit states $|0\rangle$ and $|1\rangle$, with transition frequency $\omega_{10}$. This frequency can be adjusted independently for each qubit through the bias current $I_{\text {bias }}^{\mathrm{A}, \mathrm{B}}$. Each qubit's state is detected through a single-shot measurement ${ }^{18,19}$, using a fast pulse $I_{Z}^{\mathrm{A}, \mathrm{B}}$ combined with read-out using an on-chip superconducting quantum interference device.

State preparation and tomography use single-qubit logic operations, corresponding to rotations about the $x, y$ and $z$ axes of the Bloch sphere ${ }^{19}$. Rotations about the $z$ axis are produced by fast $\left(\sim\right.$ nanosecond) current pulses $I_{Z}^{\mathrm{A}, \mathrm{B}}(t)$, which adiabatically change the qubit frequency, turning on and off the interaction and leading to phase accumulation between the $|0\rangle$ and $|1\rangle$ states. Rotations about any axis in the $x-y$ plane are produced by microwave pulses resonant with each qubit's transition frequency, applied through $I_{\mu \mathrm{w}}^{\mathrm{A}, \mathrm{B}}(t)$. The phase of the microwave pulses defines the rotation axis in the $x-y$ plane, and the pulse duration and amplitude control the rotation angle. In previous work ${ }^{20}$, such single-qubit gates were shown to have fidelities of $98 \%$, limited by the energy relaxation $T_{1}$ and dephasing $T_{2}$ times, which for this device were measured to be 400 and 120 ns, respectively. 


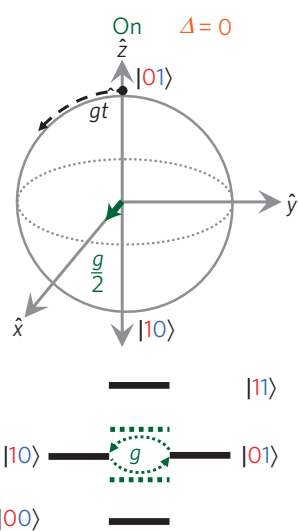

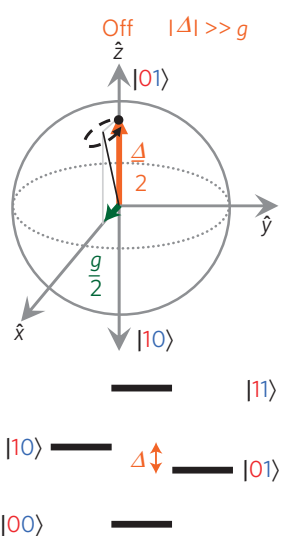

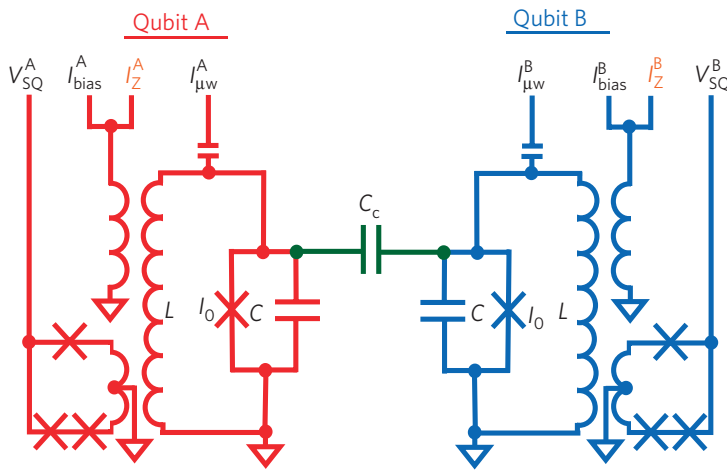

Figure 1 | Bloch-sphere, energy-level and circuit representations of the device. a,b, Energy-level diagram with coupling interaction turned on (a) and off (b). a, When qubits are on-resonance $(\Delta=0)$, their interaction swaps the populations of the $|01\rangle$ and $|10\rangle$ states at a frequency given by the coupling

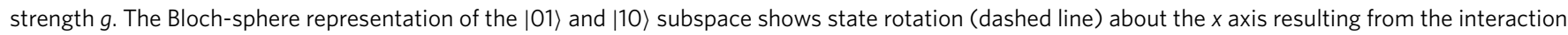

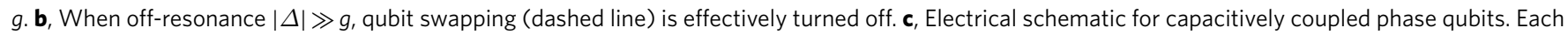
qubit junction (single cross) with critical current $I_{0}$ is shunted by an external capacitor $C$ and inductor $L$. An interdigitated capacitor $C_{c} \sim 2$ fF couples the qubits, yielding an interaction $g / 2 \pi=11 \mathrm{MHz}$. Qubit bias is through $I_{\text {bias, }}$ microwave control through $I_{\mu w}$ and qubit readout is carried out using a three-junction superconducting quantum interference device (three crosses) read out by $V_{\mathrm{SQ}}$. Fast pulses on the $I_{z}$ lines bias the qubits on- and off-resonance.

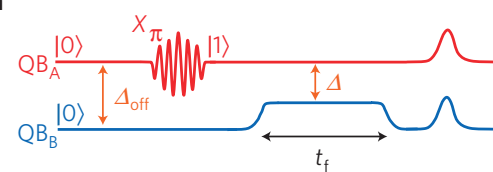

c

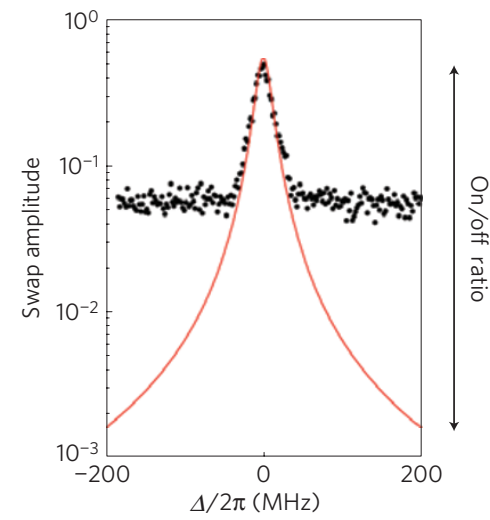

b
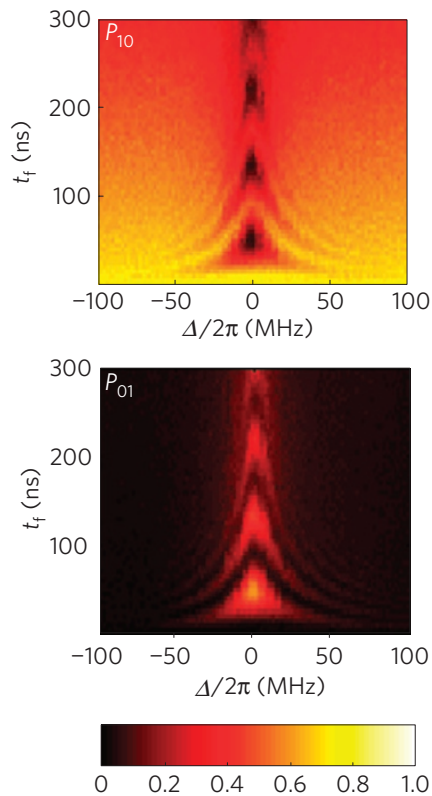

Figure 2 | Characterization of coupling interaction and measurement of on/off ratio. a, Sequence of operations: the $|10\rangle$ state is prepared by applying a 16-ns $\pi$-pulse to qubit $A$, and immediately followed by a fast pulse $I_{Z}^{B}$ that places qubit B close to resonance with qubit $A$ (detuning $\Delta$ ). After the qubits interact for a time $t_{f}$, the state occupation probabilities for each qubit are measured simultaneously. $\mathbf{b}$, Measured occupation probabilities $P_{10}$ and $P_{01}$ versus detuning $\Delta$ and interaction time $t_{\mathrm{f}}$. The oscillation period at $\Delta=0$ yields the measured coupling $g / 2 \pi=11 \mathrm{MHz}$. Data were taken up to a maximum detuning of $200 \mathrm{MHz}$, but only up to $100 \mathrm{MHz}$ is shown. The amplitude of oscillations decreases with detuning as expected. c, Peak-to-peak swapping amplitude versus detuning $\Delta$, plotted with the predicted dependence $g^{2} /\left(g^{2}+\Delta^{2}\right)$. The vertical scale for $g^{2} /\left(g^{2}+\Delta^{2}\right)$ is adjusted to match the on-resonance amplitude at $\Delta=0$. Determination of the swapping probability is limited to $>6 \times 10^{-2}$ by measurement noise. The calculated on/off ratio is indicated by the vertical arrow.

The experimental design was chosen to give qubit frequencies $\omega_{10}^{\mathrm{A}, \mathrm{B}} / 2 \pi \cong 5.5 \mathrm{GHz}$. The strength of the coupling $g=\left(C_{\mathrm{c}} / C\right) \omega_{10}^{\mathrm{A}, \mathrm{B}}$ was set by the coupling and qubit capacitances $C_{\mathrm{c}} \approx 2 \mathrm{fF}$ and $C \approx 1 \mathrm{pF}$, respectively. The coupling interaction is turned on and off by changing the relative qubit frequency $\Delta=\omega_{10}^{\mathrm{A}}-\omega_{10}^{\mathrm{B}}$ through an adjustment of the qubit B bias $I_{\text {bias }}^{\mathrm{B}}$. A large detuning of $\Delta_{\text {off }} / 2 \pi \approx 200 \mathrm{MHz}$ was used to turn off the gate, yielding a small amplitude in the off-diagonal coupling $g / \Delta_{\text {off }} \approx 0.055$.
We first characterize the coupling by measuring the time dynamics of the entangling swap operation $|01\rangle \leftrightarrow|10\rangle$ as shown in Fig. 2a. Initially, both qubits are tuned off-resonance by $200 \mathrm{MHz}$ and allowed to relax to the $|00\rangle$ state. A $\pi$ pulse on qubit A then produces the $|10\rangle$ state. A current pulse $I_{Z}^{\mathrm{B}}(t)$ applied to $I_{\text {bias }}^{\mathrm{B}}$ brings the qubits within a frequency $\Delta$ of resonance. After an interaction time $t_{\mathrm{f}}$, the bias $I_{Z}^{\mathrm{B}}$ is reset to the original $200 \mathrm{MHz}$ detuning and both qubits are then measured. Averaging 

Input QST
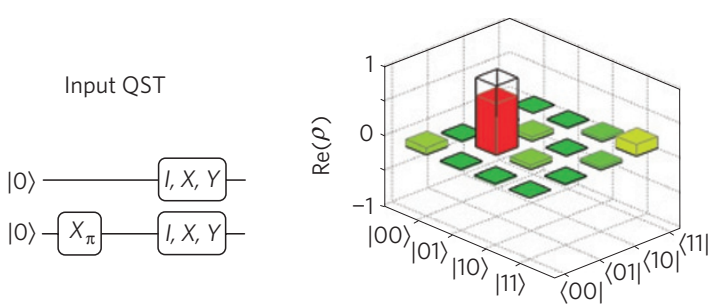

$\widehat{\overline{0}}$

Output QST
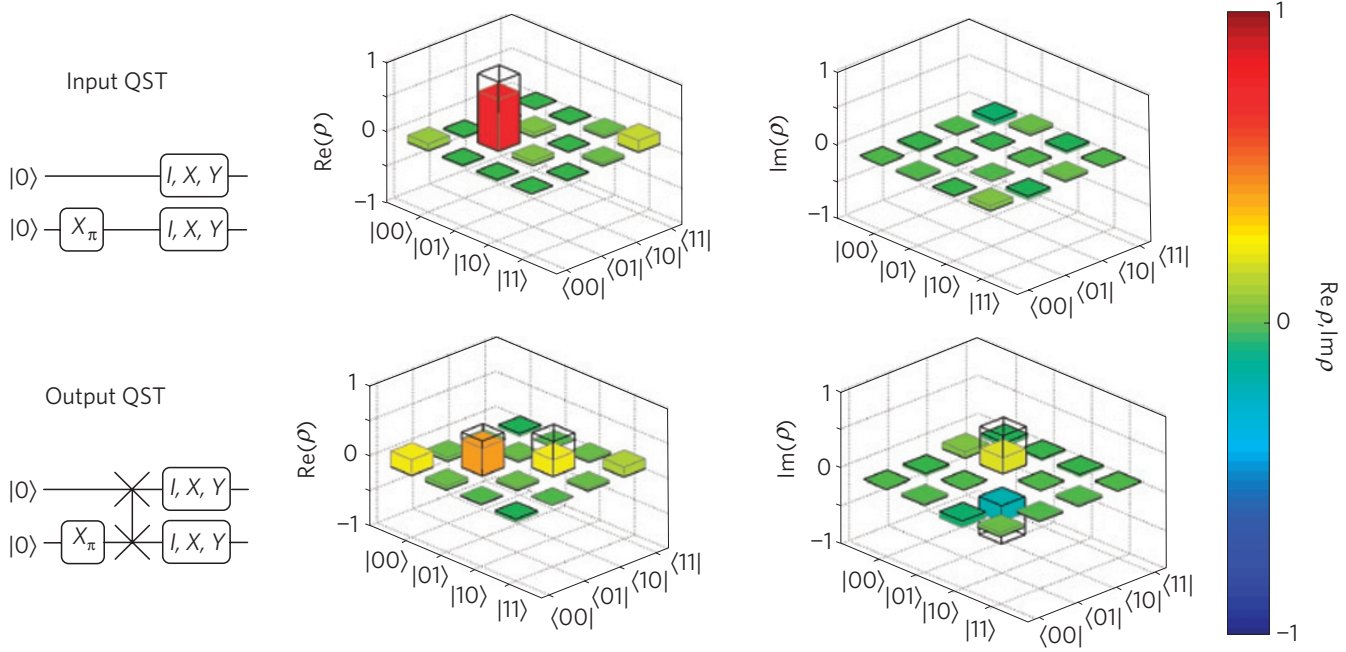

b

Input QST
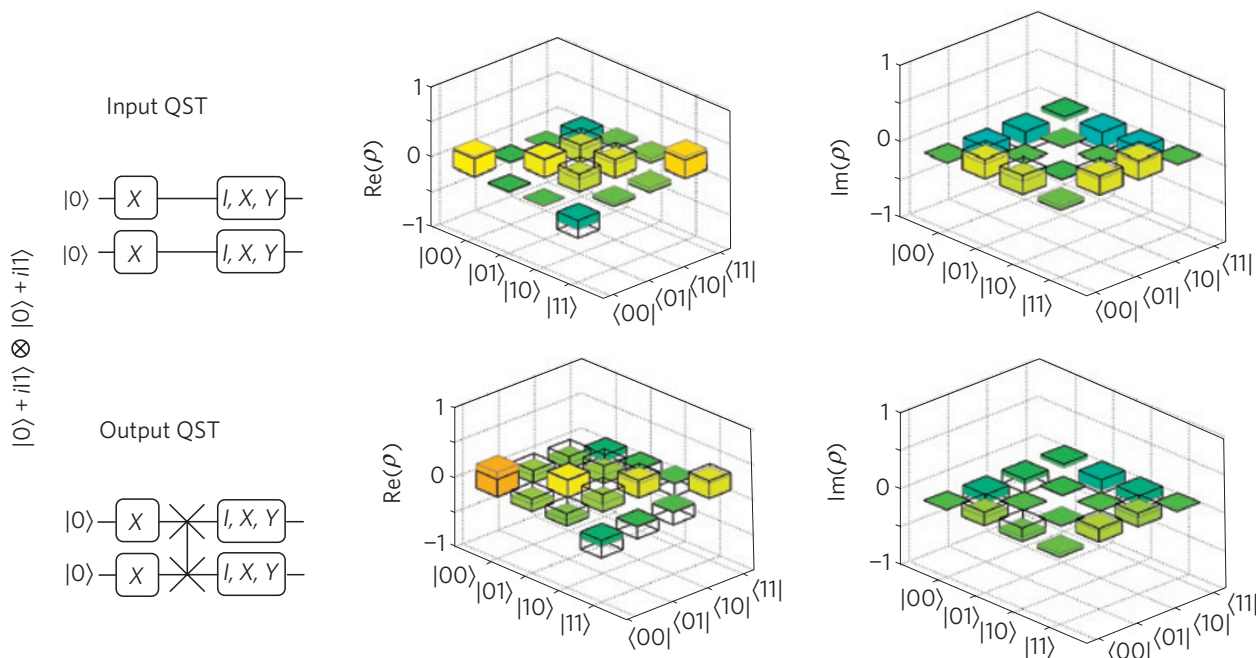

Figure 3 | Quantum state tomography for two sets of input and output states. Control sequences are shown in the left panels, where the SQiSW gate is

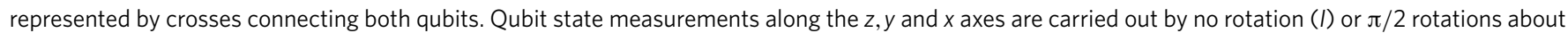

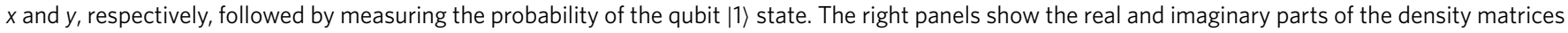
obtained in this way. The experimental data (with no corrections) and theory (with no decoherence) are shown as solid and transparent bars, respectively.

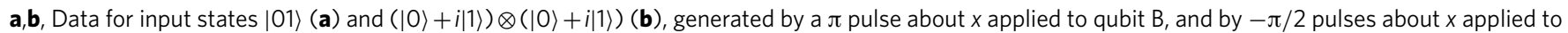
both qubits $A$ and $B$, respectively.

over 1,200 events gives the probabilities for the four possible final states $|00\rangle,|01\rangle,|10\rangle$ and $|11\rangle$. The swapping behaviour for the states $|01\rangle$ and $|10\rangle$ as a function of $t_{\mathrm{f}}$ is shown in Fig. 2b. On resonance $(\Delta=0)$, the swapping frequency between $|01\rangle$ and $|10\rangle$ gives an accurate measurement of the coupling strength $g / 2 \pi=11 \mathrm{MHz}$.

The amplitude of the swapping oscillations decreases with detuning as expected. In Fig. $2 c$ we plot the peak-to-peak change in swap probability as a function of detuning $\Delta$, compared to the theoretical prediction. Apart from a small reduction in the amplitude arising from imperfect measurement fidelity, the data is in good agreement with theory. At detunings $|\Delta| / 2 \pi>50 \mathrm{MHz}$, the swap amplitude is small and cannot be distinguished from the noise floor. From the maximum detuning bias of $\Delta / 2 \pi=200 \mathrm{MHz}$ and from the coupling strength $g / 2 \pi=11 \mathrm{MHz}$ obtained from spectroscopy measurements and the data in Fig. 2b, we compute the probability ratio $(\Delta / g)^{2}=(200 / 11)^{2}=300$ as a figure of merit for the on/off coupling ratio.
We fully characterize the SQiSW gate using QPT ${ }^{3,4}$. This involves preparing the qubits in a spanning set of input basis states, operating with the gate on this set of states, and then carrying out complete state tomography on the output. As illustrated in Fig. 3a, we first carry out quantum state tomography ${ }^{19,21}$ on the input state $|01\rangle$, which involves measuring the state along the $x, y$ and $z$ Bloch-sphere axes of each qubit, in nine separate experiments. We then operate on the $|01\rangle$ input state with SQiSW, and carry out complete state tomography on the output. These measurements allow for the evaluation of the twoqubit density matrix. This entire process is repeated 16 times in total, using four distinct input states for each qubit, chosen from the set $\{(|0\rangle,|1\rangle,|0\rangle+|1\rangle,|0\rangle+i|1\rangle)\}$. In Fig. 3b, we show the density matrix resulting from this tomography for one such input state: $(|0\rangle+i|1\rangle) \otimes(|0\rangle+i|1\rangle)$. From this complete set of measurements, we reconstruct the 16 by $16 \chi$ matrix, the indices of which correspond to the Kronecker product of the operators $\left\{I, \sigma_{x},-i \sigma_{y}, \sigma_{z}\right\}$ for each qubit ${ }^{3}$. 

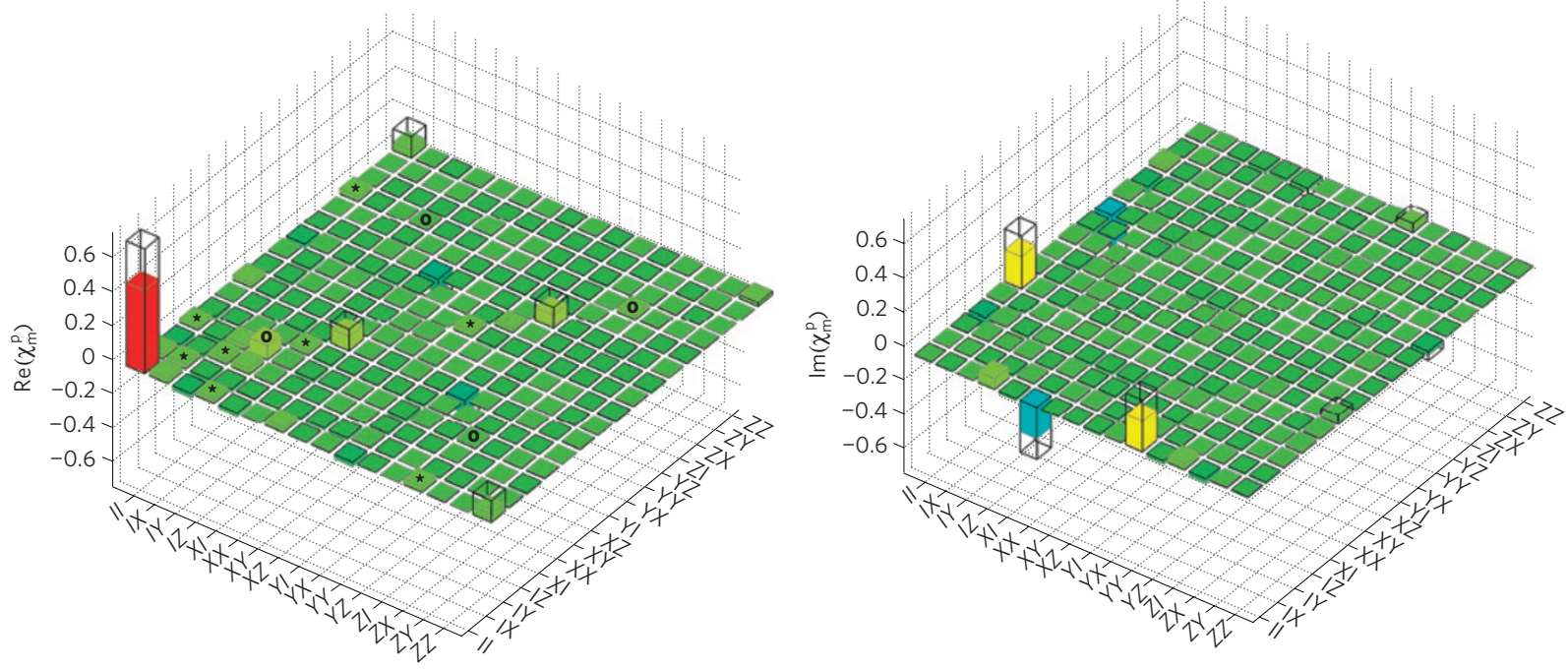

\begin{tabular}{|ccccccc|}
\hline-0.6 & -0.4 & -0.2 & 1 & 1 & 1 & \\
\hline & & $\operatorname{Re}\left(\chi_{m}^{p}\right), \operatorname{Im}\left(\chi_{m}^{p}\right)$ & & 0.4 & 0.6
\end{tabular}

Figure 4 | Real and imaginary parts of the reconstructed $\chi$ matrix for the SQiSW gate, obtained from 16 possible input states

$\{(|\mathbf{0}\rangle,|\mathbf{1}\rangle,|\mathbf{0}\rangle+|\mathbf{1}\rangle,|\mathbf{0}\rangle+\mathbf{i}|\mathbf{1}\rangle)\} \otimes\{(|\mathbf{0}\rangle,|\mathbf{1}\rangle,|\mathbf{0}\rangle+|\mathbf{1}\rangle,|\mathbf{0}\rangle+\mathbf{i}|\mathbf{1}\rangle)\}$. Physical estimates to the experimental data are shown as solid bars. The transparent bars give the theoretically expected $\chi$ matrix, which does not include effects resulting from decoherence. Calibrations from measurement were accounted for in this analysis. Technical details regarding calibrations and the physical estimation can be found in the Supplementary Information. The matrix elements of $\chi_{\mathrm{m}}^{\mathrm{p}}$ that are non-zero because of energy relaxation and dephasing are marked with an asterisk and a circle, respectively.

In a QPT experiment ${ }^{22-24}$ errors arise from the entangling gate and errors in measurement. As we are interested in the quality of the entangling gate itself, we have calibrated out errors resulting from measurement ${ }^{18}$. As described in the Supplementary Information, measurement errors arise from both a misidentification of the $|0\rangle$ and $|1\rangle$ states, and measurement crosstalk, where a measurement of $|1\rangle$ in one qubit increases the probability of a $|1\rangle$ measurement in the second qubit ${ }^{18}$. By carrying out further calibration experiments, we are able to determine the probabilities for these errors and correct the probabilities of the $|00\rangle,|01\rangle,|10\rangle$ and $|11\rangle$ final states.

In addition, standard QPT typically produces an unphysical $\chi$ matrix because of inherent experimental noise ${ }^{22,24}$. A physical $\chi$ matrix must be completely positive and trace preserving ${ }^{3}$ (CPTP), which implies it must have positive eigenvalues that sum to one. Our measured $\chi$ matrix has several negative eigenvalues, as discussed in the Supplementary Information. As is commonly done in QPT experiments ${ }^{22,24}$, a $\chi$ matrix that satisfies the CPTP constraints must be obtained from the experimental data before the data can be compared to a theoretically predicted $\chi$ matrix, which is physical by construction. This can be computed by noting that QPT is essentially analogous to system identification from classical control theory. More specifically, the problem of finding a physical approximation to unphysical QPT data can be shown to be a convex optimization problem ${ }^{25,26}$, a technique commonly used in control theory. We use a type of convex optimization called semidefinite programming ${ }^{25}$ to find the physical $\chi$ matrix that best approximates our measured, unphysical $\chi$ matrix. Mathematically, for the experimentally obtained $\chi$ matrix and the physical approximation $\chi^{\mathrm{p}}$, we minimize the two-norm distance $\left\|\chi^{\mathrm{p}}-\chi\right\|_{2} \equiv \sqrt{\operatorname{tr}\left\{\left(\chi^{\mathrm{p}}-\chi\right)^{2}\right\}}$ with the constraints that $\chi^{\mathrm{p}}$ be CPTP.

This physical matrix $\chi_{\mathrm{m}}^{\mathrm{p}}$, which also includes the calibrations for measurement errors, is shown in Fig. 4. This matrix closely matches the original data before corrections for CPTP (see Supplementary Information). In both the real and imaginary parts of the $\chi_{\mathrm{m}}^{\mathrm{p}}$ matrix, we observe non-zero matrix elements in locations where such elements are expected, in qualitative agreement with the theory, shown as the transparent bars. Quantitative comparison is obtained by calculating the process fidelity, $0<F_{\mathrm{p}}<1$, which gives a measure of how close $\chi_{\mathrm{m}}^{\mathrm{p}}$ is to theoretical expectations ${ }^{24}$. For the SQiSW gate demonstrated here, with measurement calibration taken into account, we find $F_{\mathrm{p}}=\operatorname{Tr}\left(\chi_{\mathrm{t}} \chi_{\mathrm{m}}^{\mathrm{p}}\right)=0.63$, where $\chi_{\mathrm{t}}$ is the theoretical $\chi$ matrix for the SQiSW gate. $\chi_{\mathrm{m}}$ and $\chi_{\mathrm{e}}$, the unphysical $\chi$ matrices with and without measurement calibrations, respectively, and $\chi_{\mathrm{e}}^{\mathrm{p}}$, the physical approximation to the $\chi$ matrix that does not include the measurement calibrations, are shown in Supplementary Figs S1-S3.

Errors in our SQiSW gate primarily arise because the time for the experiment ( $\sim 50 \mathrm{~ns})$ is not significantly shorter than the $T_{2}$ dephasing time of $120 \mathrm{~ns}$. This is confirmed using a recent theory ${ }^{5}$ by Kofman et al., which includes the effects of dephasing and decoherence on the SQiSW $\chi$ matrix. In particular, the elements marked with an asterisk and a circle in Fig. 4 are non-zero because of energy relaxation and dephasing, respectively. The modified Pauli basis used here gives a sign change in the real part of $\left(\chi_{\mathrm{m}}^{\mathrm{p}}\right)_{X X, Y Y}$ and $\left(\chi_{\mathrm{m}}^{\mathrm{p}}\right)_{Y Y, X X}$, and the imaginary part of $\left(\chi_{\mathrm{m}}^{\mathrm{p}}\right)_{X X, I I}$ and $\left(\chi_{\mathrm{m}}^{\mathrm{p}}\right)_{I I, X X}$. Using this theory and the real part of $\left(\chi_{\mathrm{m}}^{\mathrm{p}}\right)_{I Z, I Z}$ and $\left(\chi_{\mathrm{m}}^{\mathrm{p}}\right)_{I Z, Z I}$, we estimate our single-qubit dephasing time as $T_{2}=(3 \pi+2) / 16 g\left(\chi_{\mathrm{m}}^{\mathrm{p}}\right)_{I Z, I Z}$. From Fig. 4 we find $\left(\chi_{\mathrm{m}}^{\mathrm{p}}\right)_{I Z, I Z}=0.105$ and $T_{2}=123 \mathrm{~ns}$, in close agreement with the value mentioned above obtained from Ramsey experiments. We also estimate the degree of correlation of the dephasing noise between the coupled qubits using $\kappa \approx\left(\chi_{\mathrm{m}}^{\mathrm{p}}\right)_{I Z, Z I} /\left(\chi_{\mathrm{m}}^{\mathrm{p}}\right)_{I Z, I Z}-[(\pi-2) /(3 \pi+2)]$. Our measurement of $\left(\chi_{\mathrm{m}}^{\mathrm{p}}\right)_{I Z, Z I} \approx 0.017$ yields $\kappa \cong 0.11$, indicating that the dephasing is mostly uncorrelated. This is in agreement with previous work ${ }^{27-29}$ that found a dephasing mechanism local to the individual qubits.

Received 9 June 2009; accepted 3 March 2010; published online 25 April 2010

\section{References}

1. Plantenberg, J. H., de Groot, P. C., Harmans, C. J. \& Mooij, J. E. Demonstration of controlled-NOT quantum gates on a pair of superconducting quantum bits. Nature 447, 836-839 (2007). 
2. Yamamoto, Y., Pashkin, Y. A., Astafiev, O., Nakamura, Y. \& Tsai, J. S. Demonstration of conditional gate operation using superconducting charge qubits. Nature 425, 941-944 (2003).

3. Chuang, I. L. \& Nielsen, M. A. Prescription for experimental determination of the dynamics of a quantum black box. J. Mod. Opt. 44, 2455-2467 (1997).

4. White, A. G. et al. Measuring controlled-NOT and two-qubit gate operation. J. Opt. Soc. Am. B 24, 172-183 (2007)

5. Kofman, A. G. \& Korotkov, A. N. Two-qubit decoherence mechanisms revealed via quantum process tomography. Phys. Rev. A 80, 042103 (2009).

6. Wolf, M. M., Eisert, J., Cubitt, T. S. \& Cirac, J. I. Assessing non-markovian quantum dynamics. Phys. Rev. Lett. 101, 150402 (2008).

7. Kosut, R. L. Quantum process tomography via L1-norm minimization. Preprint at http://arxiv.org/abs/0812.4323 (2009).

8. Mohseni, M., Rezakhani, A. T. \& Lidar, D. A. Quantum-process tomography: Resource analysis of different strategies. Phys. Rev. A 77, $032322(2008)$.

9. Howard, M. Quantum process tomography and Lindblad estimation of a solid-state qubit. New J. Phys. 8, 33 (2006).

10. Chow, J. M. et al. Randomized benchmarking and process tomography for gate errors in a solid-state qubit. Phys. Rev. Lett. 102, 090502 (2009).

11. Neeley, M. et al. Process tomography of quantum memory in a Josephson-phase qubit coupled to a two-level state. Nature Phys. 4, 523-526 (2008).

12. Bremner, M. J. et al. Practical scheme for quantum computation with any two-qubit entangling gate. Phys. Rev. Lett 89, 247902 (2002).

13. Schuch, N. \& Siewert, J. Natural two-qubit gate for quantum computation using the XY interaction. Phys. Rev. A 67, 032301 (2003).

14. Wendin, G. \& Shumeiko, V. S. Superconducting quantum circuits, qubits and computing. Preprint at http://arxiv.org/abs/cond-mat/0508729 (2005).

15. Geller, M. R. et al. NATO Science Series II: Mathematics, Physics and Chemistry: Quantum Computing With Superconductors I: Architectures 171-194 (Springer, 2007).

16. You, J. Q. \& Nori, F. Superconducting circuits and quantum information. Phys. Today 58, 42-47 (2005).

17. Devoret, M. H. \& Martinis, J. M. Implementing qubits with superconducting integrated circuits. Quantum Inform. Process 3, 163-203 (2004).

18. McDermott, R. et al. Simultaneous state measurement of coupled Josephson phase qubits. Science 307, 1299-1302 (2005).

19. Steffen, M. et al. Measurement of the entanglement of two superconducting qubits via state tomography. Science 313, 1423-1425 (2006).

20. Lucero, E. et al. High-fidelity gates in a single Josephson qubit. Phys. Rev. Lett. 100, 247001 (2008).
21. Liu, Y., Wei, L. F. \& Nori, F. Quantum tomography for solid-state qubits. Europhys. Lett. 67, 874-880 (2004).

22. Riebe, M. et al. Process tomography of ion trap quantum gates. Phys. Rev. Lett. 97, 220407 (2006).

23. Childs, A. M., Chuang, I. L. \& Leung, D. W. Realization of quantum process tomography in NMR. Phys. Rev. A 64, 012314 (2001).

24. O'Brien, J. L. et al. Quantum process tomography of a controlled-not gate. Phys. Rev. Lett. 93, 080502 (2004).

25. Boyd, S. \& Vandenberghe, L. Convex Optimization (Cambridge Univ. Press, 2004).

26. Branderhorst, M. P. A., Nunn, J., Walmsley, I. A. \& Kosut, R. L. Simplified quantum process tomography. New J. Phys. 11, 115010 (2009).

27. Sendelbach, S. et al. Magnetism in SQUIDs at millikelvin temperatures. Phys. Rev. Lett. 100, 227006 (2008).

28. Bialczak, R. C. et al. $1 / f$ flux noise in Josephson phase qubits. Phys. Rev. Lett. 99, 187006 (2007)

29. McDermott, R. Materials origins of decoherence in superconducting qubits. IEEE Trans. Appl. Supercond. 19, 2-13 (2009).

\section{Acknowledgements}

Devices were made at the UCSB Nanofabrication Facility, a part of the NSF-funded National Nanotechnology Infrastructure Network. We thank A. N. Korotkov for discussions on parameter extraction from the $\chi$ matrix and J. Eisert for assistance on using convex optimization to approximate a physical $\chi$ matrix. Semidefinite programming convex optimization was carried out using the open-source MATLAB packages YALMIP and SeDuMi. This work was supported by IARPA (grant W911NF-04-1-0204) and by the NSF (grant CCF-0507227).

\section{Author contributions}

R.C.B. designed and fabricated the samples, designed and carried out experiments and analysed the data. R.C.B. co-wrote the paper with J.M.M. and A.N.C., who also supervised the project. M.A. and M.N. provided assistance with data-taking software. E.L. and M.H. provided assistance with data-taking electronics. M.S. provided assistance with data analysis. All authors contributed to experiment set-up, sample design or sample fabrication.

\section{Additional information}

The authors declare no competing financial interests. Supplementary information accompanies this paper on www.nature.com/naturephysics. Reprints and permissions information is available online at http://npg.nature.com/reprintsandpermissions. Correspondence and requests for materials should be addressed to J.M.M. 